\title{
A Review on Prognostics and Health Management
}

\author{
Nannan $\mathrm{Hu}^{\mathrm{a}}$, Xiaohao $\mathrm{Su}^{\mathrm{b}}$ and Baolong $\mathrm{Liu}^{\mathrm{c}}$ \\ School of Computer Science and Engineering \\ Xi'an Technological University \\ Xi'an, 710021, China \\ a e-mail: 1257275813@qq.com \\ b e-mail: su-xiaohao@foxmail.com \\ c e-mail: liu.bao.long@hotmail.com
}

\begin{abstract}
The health management of complex equipment is crucial to ensure the reliability, maintainability and safety of complex equipment. This paper introduces the basic concept and research connotation of prognostics and health management (PHM), analyzes the significance of PHM technology in equipment maintenance. For the fault prediction architecture and method, this paper focuses on the discussion, analysis, and summarizes the current research's hot spots and existing technical difficulties. Finally, the future research directions are also prospected.
\end{abstract}

Keywords-Fault Prognostics; Health Management; Fault Diagnosis; Remaining Life

\section{INTRODUCTION}

With the rapid development of modern technologies, especially information technologies, the complexity, integration, and intelligence of a large number of complex systems are constantly increasing, and the costs of research, production, and especially maintenance and protection are increasing. At the same time, due to the increase of components and influence factors, the probability of failure and function failure gradually increases. Therefore, the fault diagnosis and maintenance of complex systems gradually become the focus of researchers. PHM [1-2] is gaining more and more attention.

The concept of PHM first appeared in military equipment, obtained applications in complex systems and equipment such as spacecraft, aircraft and nuclear reactors. With the continuous development of PHM technology, PHM has been gradually focused by many industrial fields in electronics, automobile, ships, the safety of engineering structures and other applications are also increasing [3-5]. The PHM is a further extension of the build-in test (BIT) and state's health monitoring capabilities used for complex systems. It is a shift from state monitoring to health management, to identify and manage the occurrence of faults, planning maintenance and supply assurance. The main purpose is to reduce the maintenance costs, improve equipment system security and tasks' success, achieve condition-based maintenance [6] (CBM) and autonomous protection with less maintenance inputs.

\section{CONNOTATION OF PHM}

\section{A. The Basic Concept of PHM}

PHM contains two aspects, fault prognostics and health management. Health refers to the performance's degradation or deviation compared with the expected normal performance state. Failure prognostics refers to the prediction based on the current or historical performance state of the system, diagnose its function, including determining the remaining life of the system or the length of time it is expected to work; Health management is a ability to make appropriate decisions, based on diagnostic, prognostic information, available maintenance resources and usage requirements.

PHM represents a shift in approach, in maintenance strategy and conceptual, that enables a shift from traditional sensorbased diagnostics to prognostics based on intelligent systems, to provide accurate technical support at the right time. PHM technology also makes after-hours maintenance or regular maintenance strategies are replaced by the situation of maintenance. This change can bring the following improvements to the actual equipment protection [7]:

- Provide senior alarm of system failure.

- Provide condition-based maintenance ability.

- Improve the availability of the system through maintenance cycle extension.

- Reduce the cost of full life cycle by reducing inspection cost and failure time.

- Reduce the occurrence of intermittent and no fault founds (NFF).

\section{B. Connotation of PHM}

In system function [8], PHM can achieve state monitoring, fault detection, failure prediction, the remaining life prognostics of key components or system; It is a combination of multiple frontiers and Interdisciplinary. In the evolution of technology, it is the improvement of traditional equipment condition monitoring and fault diagnosis, emphasizing the discovery of equipment failure before the failure of early signs, tracking the development of fault symptoms while assessing the remaining useful life of the equipment, providing decision's support for the maintenance of the equipment ultimately.

The health degradation process of a device is a process in which the device's health status changes from normal to degressive until the function fails. Equipment health degradation process is shown in [9-10] Fig. 1. 


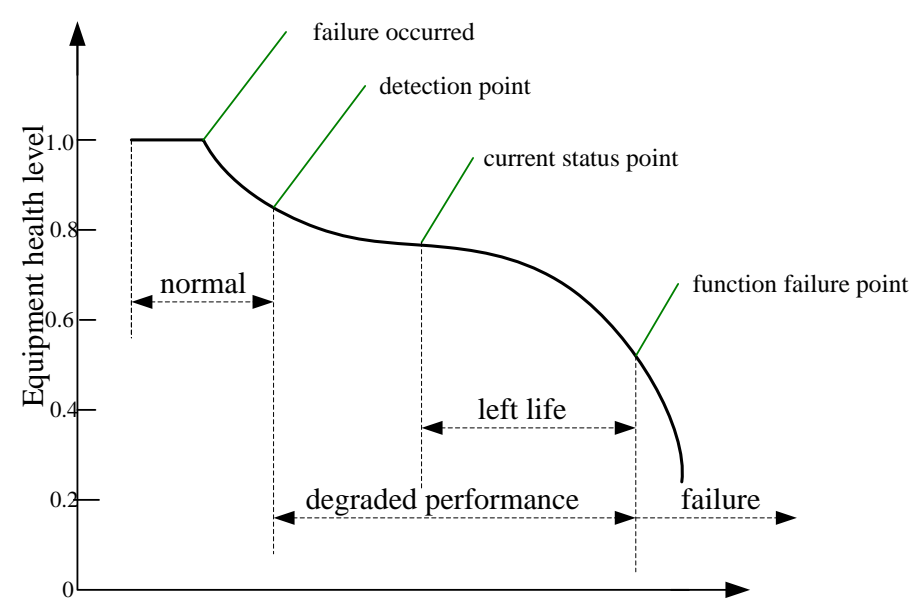

Figure 1. Equipment health degradation process.

In view of the above typical equipment health degradation process, the fault system should have early fault detection capabilities, and monitor its degradation process. Fault prognostics research includes the following aspects:

- Evaluate which health condition the current device is in its health degradation process: a normal state, a degraded performance state, or a functional failure state.

- When the equipment is in a state of degraded performance, judging what kind of failure mode which causes its health level to drop, and evaluating the current state of health deviation from its normal state.
- Predicting the future state of health of equipment, it has two forms: (1) Study whether the equipment can complete its functional requirements normally in a period of time in the future (2) Study the remaining life of equipment

\section{SySTEM APPLICATION OF PHM}

\section{A. PHM's Framework}

PHM systems generally should be capable of fault detection, fault isolation, enhanced diagnostics, performance testing, fault prognostic, health management, and component life tracking [11]. Most fault diagnosis and fault prognostic tools have domain-related features [12-13]. The PHM technical system framework is shown in Fig. 2.

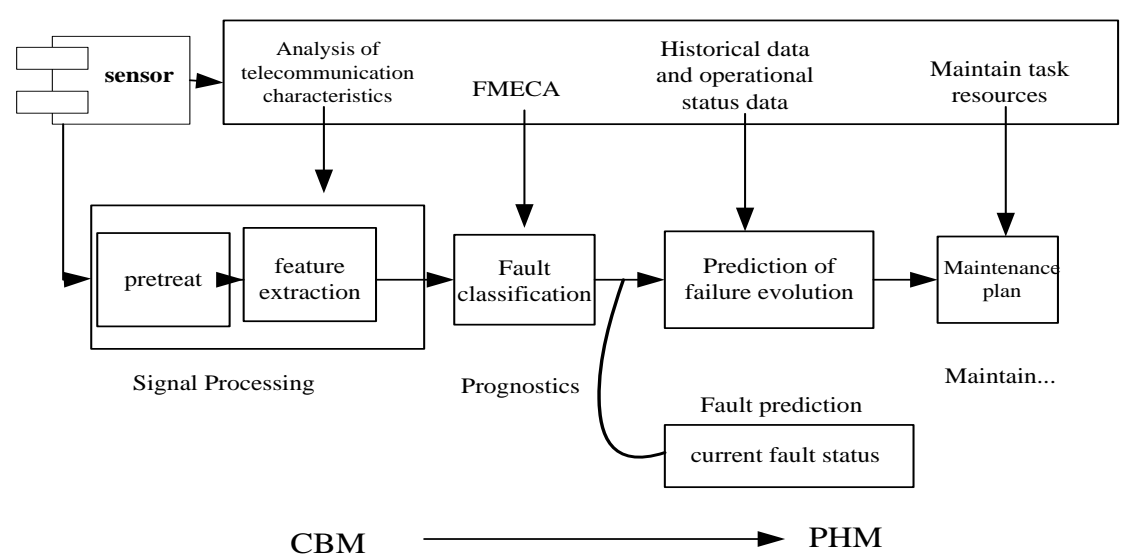

Figure 2. Framework for PHM.

The method system of PHM technology is shown in Fig.3. Firstly, evaluating the virtual life; Through the input of design data, expected life cycle operating conditions, failure modes and failure impact analysis (FMMEA) and physics-of-failure (PoFs) models, etc., to achieve reliability (or virtual life) evaluation.

FMECA is of great significance in the selection of design schemes, objective evaluation of relevant measures (such as unnecessary measures) and testing equipment, and at the same 
time. In order to apply it to the design of fault prognostics and state management, the following parts should be added to the traditional FMEA: the signs and symptoms before the failure mode occurs; the signs that the product or function may have been severely damaged; the signs of the failure mode are observed or the location of sensors; fault detection methods and fault prognostic methods [15].

During FMECA analysis, when analyzing the cause of fault, it is also necessary to analyze the potential source of fault in depth, emphasizing the relationship between other products or external factors in the state of each other. If FMEA analysis table further analyzes the "root cause of the fault" for the cause of the fault, it firstly analyzes the root fault mode, and identifies external manifestation of the root cause, and its functional impact on the subsystem or equipment. If there is no problem (such as temperature, degree of vacuum, normal pressure, etc.) through the detection or monitor, then it is no longer necessary to take the FMEA analysis for the root cause of fault.

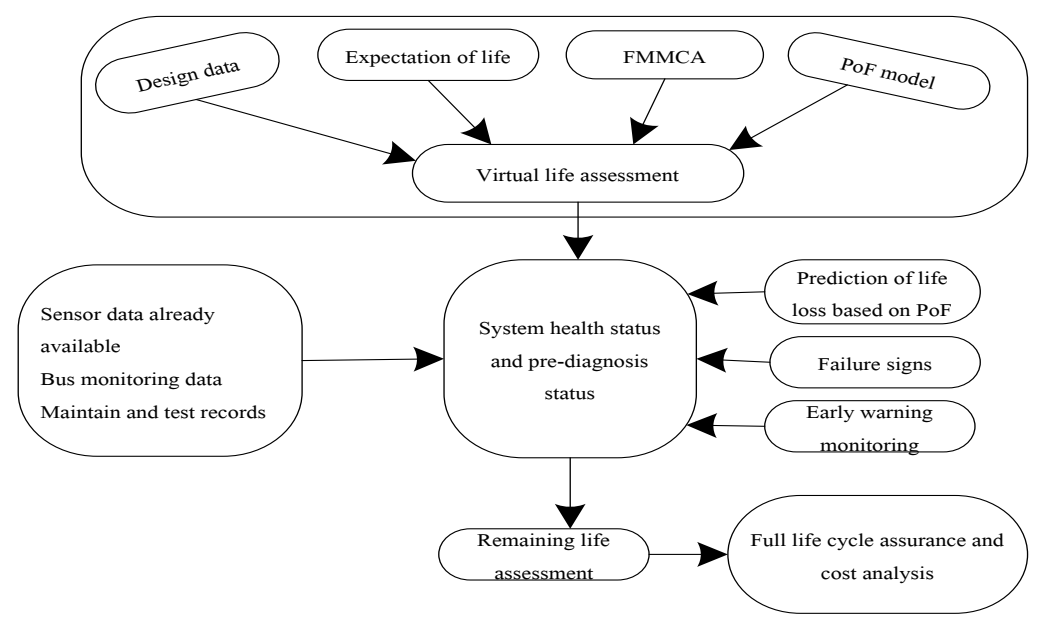

Figure 3. General methodology of PHM [11].

By performing a virtual life assessment, the major failure modes and failure mechanisms to be prioritized can be determined. Existing sensor data, monitoring data, maintenance and inspection records can also be used to identify abnormal conditions and parameters. In the entire methodological system, prediction is the core method and research content to achieve system performance degradation status and remaining life prediction.

\section{B. Fault prognostic model design of PHM}

PHM is a new system maintenance and management philosophy that significantly improves the understanding of the behavior of complex systems through comprehensive

In order to accurately predict a fault before it occurs, it is necessary to capture the symptom of the fault by monitoring the change of the system state parameter. For a slowly changing fault, before the near failure occurs, the rate of change of the system state parameter usually increases sharply. Therefore, it is the key to successfully predicting failures by reasonably determining the critical point of state change that is about to occur.

First, based on the results of signal analysis, the analysis of the root cause of the failure, and the extension of FMECA results, the correlation equations between the model parameters $\theta_{i}$ (describe the overall state of the system) and the physical parameters $P_{j}$ (describe subsystems and part properties) is established as follows: fault detection, isolation and prognostic and state management [16]. At the same time, information on key components is also collected and processed to prognostic the remaining useful life. The realization of fault prognostics mainly depends on the model design. In view of the complicated system of PHM, it is urgent to develop and design a general prognostic framework to select and integrate the appropriate methods of diagnosis and prediction. Improve the utilization of information, and achieve multi-angle and multi-parameter prognostic, improve the level of diagnosis and prognostics [17].

$$
\theta_{i}=f\left(P_{j}\right)
$$

Estimating signal input and output parameter from the measurement, the estimated model parameter $\theta_{i}$ to calculate the actual physical parameters:

$$
P=f^{-1}\left(\theta_{i}\right)
$$

Determine the change of the actual physical parameter $P_{0}$ relative to the nominal physical parameter value $\Delta P$ [18]. $\Delta P$ is a reflection of the operational status of the equipment, whether the system is abnormal operating status, the size of the anomalies. They can be real-time described by $\Delta P$ 's value and changes. When setting up the correlation equation $\theta_{i}=f\left(P_{j}\right)$, the limit value should be 
set to the value at the performance of the system suddenly

Observe the value of $\Delta P$ in real time, according to the changes of $\Delta P$ (whether exceed the critical value), as well as changes in the physical parameters, the corresponding relationship between the decision-making, to determine whether the failure will occur. Finally, the use of statistical decisions or hypothesis testing, fault separation, to determine the impending failure causes, size and type [19].

In addition, after the fault symptoms have been identified, fault trends should be analyzed to quantify the time since the device state was abruptly changed to the time when fault to occur. Fault trends are closely related to the material properties, design, and structural characteristics of the equipment or component. So the speed and trend of fault development can be described by computer simulation. The relationship between the remaining life of the system and the running status and running time can finally be expressed in the form of a three-dimensional surface. Fault prognostics output characteristic surface simulate as Fig. 4. The x-axis represents time, the $\mathrm{y}$-axis represents $\Delta P$ that reflects the working status, and the $\mathrm{z}$-axis represents the remaining life of the part or system. This figure can intuitively and accurately represent the remaining life of any part or system that corresponds to any operating state and any operating time point. begins to change drastically.

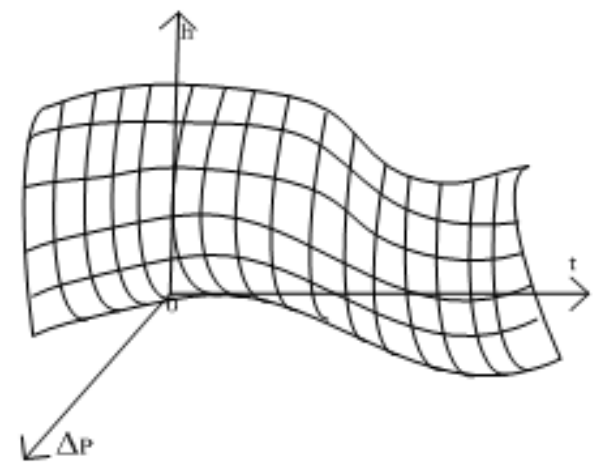

Figure 4. Fault prediction output characteristic surface map.

\section{Algorithm OF FAULt PROGNOSTIC}

According to the theories, methods and technical routes applied in the actual research, the fault prognostics technology can be divided into three types [20-25]: As shown in Fig. 5 below: The prognostic techniques are divided into three categories: (1) model-driven fault prognostics techniques; (2) data-driven fault prognostics techniques; (3) fault prognostics techniques based on statistical reliability (probability-based); The three methods in the engineering application of the extensive weakened in order, but the prognostics accuracy risen, the difficulty and cost associated with it also increased [26-28]. The following is a brief introduction to all kinds of forecasting methods.

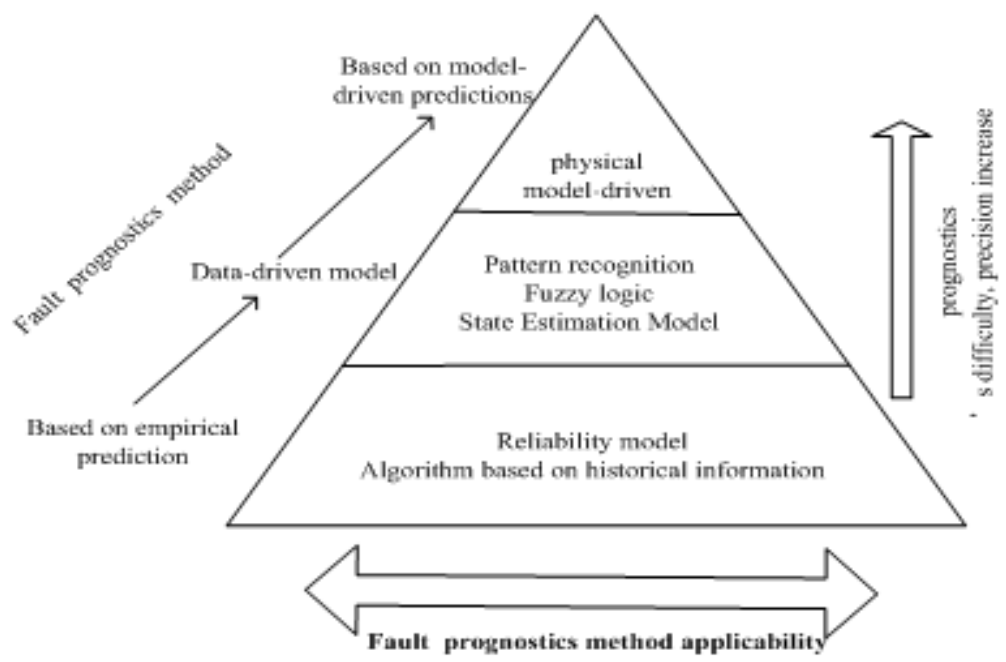

Figure 5. Algorithms of fault prognostics..

\section{A. Model-driven Fault Pragnostics Techniques}

Model-driven fault prognostic is a technique using either a dynamic model or process prognostics method. Physical model approach, Kalman/Extended Kalman filter/particle filter and expert-based methods can be classified in Model-driven fault prognostics [26]. Model- based fault prognostics techniques generally require the mathematical models of the object system to be known.

Such methods provide a means of grasping the failure mode process of the component or system being predicted. By calculating the functional impairment under system operating conditions, to assess the loss of critical components and to assess the cumulative effects of failures in the use of the 
components over the life, to evaluate the remaining useful life by integrating physical models with stochastic process modeling distribution. Model-based fault prognostics techniques have the property of being able to penetrate the nature of the object system and the advantages of real-time.

Adams [29] put forward the damage accumulation model of the first/second order nonlinear differential equation in the structural dynamic system. Chelidze [30] expressed the model of the performance degradation in the slow process; the process corresponds with time-varying. The model is used to track battery degradation (voltage). In [31], a non-linear stochastic model is proposed to model the mechanical structure. The model uses the generalized Kalman filter to estimate the current fault condition of the system in real time and predict the remaining life of the system. The literature [32-33] introduced estimating the residual life by estimating the two defect propagation models for a bearing-loaded mechanical model. Luo [34] proposed a data-based synthetic forecasting process using model-based simulation data under nominal and degraded conditions.

At present, most of the model-based methods are used in electromechanical systems such as aircraft and rotating machinery. For complex electronic systems, because of failure mechanisms are relatively complicated, so the modeling of fault prognostic lags behind.

\section{B. Data-driven Fault Pragnostics Techniques}

Compared with regression analysis and time series analysis [36] in the traditional statistical category, neural network is one of the most used methods in fault prognostic methods and applications. Unlike the modelbased methods, neural networks are based on data-driven approaches that allow data to be self-adaptive can learn from samples, and try to capture intrinsic functional relationships between sample data. Zhang and Ganesan [35] applied a self-organizing neural network to multivariate trend forecasting and applied to the prognostic of the remaining life of the bearing system. Reference [36] used recurrent neural networks (RNN) to predict system fault trends. And as the research progresses, many improved or special forms of neural network algorithms have emerged, such as wavelet neural networks (WNN), fuzzy neural networks (FNN), etc. These improved neural network algorithms have also achieved good results in fault diagnosis and prognostic.
The data-driven fault prognostic technology does not need the prior knowledge (mathematical model and expert experience) of the object system, and based on the collected data, the hidden information can be obtained through various data analysis and processing methods to prognosticate the operation. Thus, The shortcomings of the model and knowledge-based fault prognostics technology have become a more practical fault prognostics method. However, typical data (historical work data, fault injection data, and simulation experiment data) for some of the key devices in practice are usually very expensive to obtain; And even they are often highly uncertain and incompleteness, these problems all increase the difficulty of realizing the fault prognostic technology.

\section{Fault Pragnostics Techniques Based on Statistical Reliability}

This method requires less detail than model-based methods, because the information needed for the prognostic is contained in a series of different probability density functions (PDFs), without the need for dynamic differential equations. The advantage of this approach is that the required PDF can be obtained by analyzing the statistics, and the resulting PDF can provide sufficient support for the prognostic. In addition, the prognostic given by this method contains the confidence level, which can also well characterize the prognostic result.

The typical fault probability curve based on statistical reliability is the famous "bathtub curve", as shown in Fig 1. That is, the failure rate is relatively high at the beginning of the operation of the equipment or system. After a period of stable operation, the failure rate can generally be maintained at a relatively low level, and then, after a period of operation, the failure rate starts to increase again, until all parts or equipment have failed. Equipment production characteristics, historical changes, performance degradation and other life-cycle factors make the fault prognostic based on system characteristics more complicated. All of these factors will have a certain probability impact on the prognostic results. Also need to consider reducing fault prognostic false alarm rate.

\section{The Comparison of Fault Prognostic Algorithm}

Through the literature to research, the commonly used prognostic algorithms are summarized, as shown in Table 1: 
TABLE I. ALGORITHMS OF FAULT PROGNOSTICS

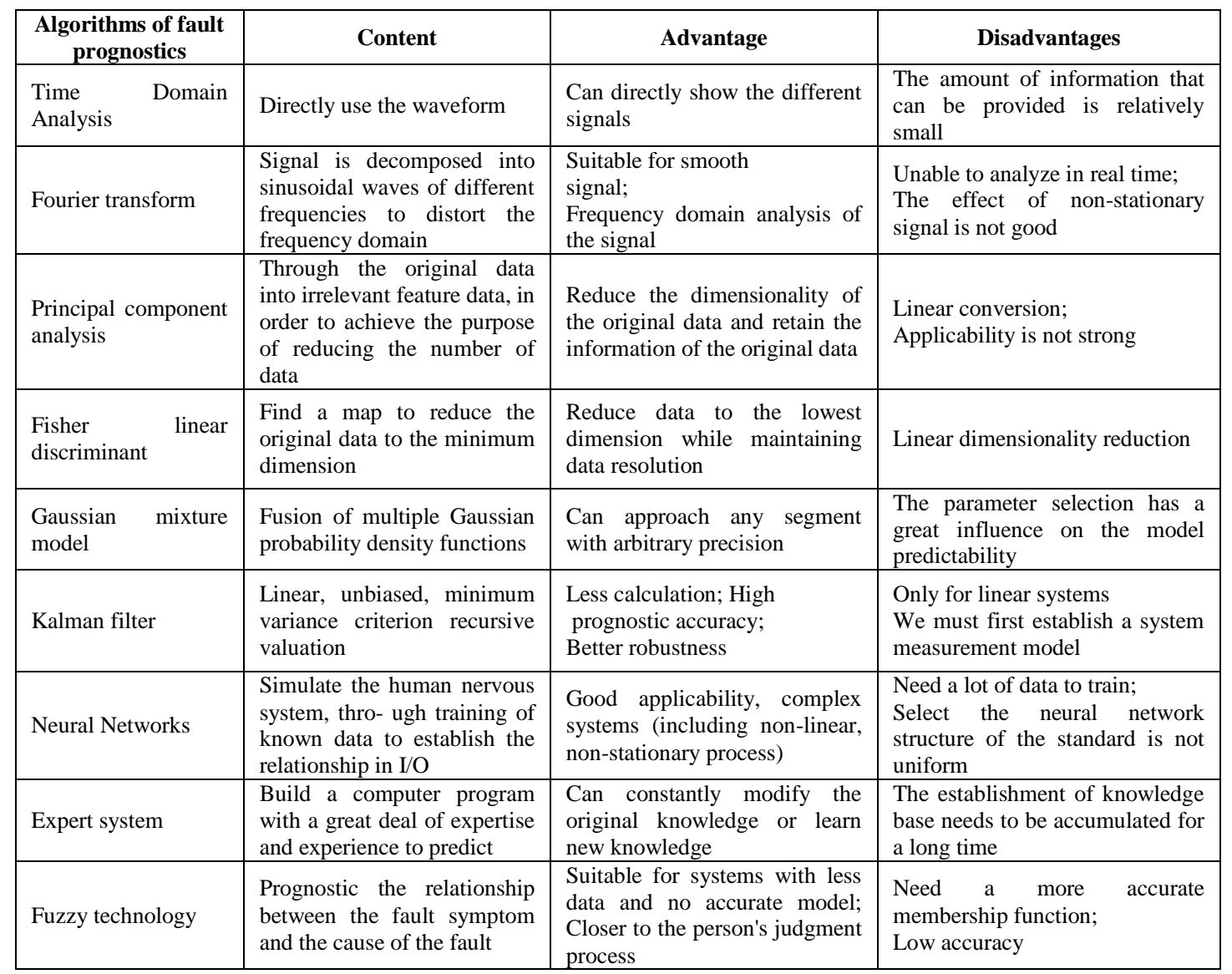

\section{CONCLUSION}

At present, China's national science and technology industry has a strong demand for PHM technology. Drawing on and absorbing foreign advanced experience, the study of PHM key technologies can provide the basic technical reserve for the development of a new generation of weapons and equipment in our country, lay the foundation for engineering application and better promote the rapid development of China's national industry. There are following five aspects should be hard work.

The process of fault prediction is often uncertain. Up to now, there is no general complex system in China. It is of great significance to our national defense industry and economic development. This paper summarizes the basic concepts, research connotation and basic research status of PHM by drawing from foreign advanced experience and deeply studying the key technologies of PHM. It mainly summarizes the main methods of PHM architecture and fault prognostic. Improve the specific technology, used in system development, will be the next major direction of research.

\section{ACKNOWLEDGMENT}

This work is partially supported by Science \& Technology Program of Shaanxi Province with project
"2017GY-196" and "2015KTCXSF-10-11", and the Opening Fund of State and Local Engineering Laboratory of Advanced Network and Monitoring Control with project "GSYSJ2016001".

\section{REFERENCES}

[1] HESS A, FILA L. The joint strike fighter (JSF) PHM Concept: Potential impact on aging aircraft problems[C]. Proceedings of IEEE Aerospace Conference, Big Sky, Montana, USA, 2002, 6: 3021-3026.

[2] KEITH M J, RAYMOND R B. Diagnostics to Prognostics A product availability technology evolution[C]. The 53rd Annual Reliability and Maintainability Symposium(RAMS 2007), Orlando, FL, USA, 2007: 113-118.

[3] NISHAD P, DIGANTA D, GOEBEL K, et al. Identification of Failure Precursor Parameters for Insulated Gate Bipolar Transistors (IGBTs)[C]. 2008 International Conference on Prognostics and Health Manageme- nt(PHM 2008), Denver, CO, USA, 2008: 1-5.

[4] HAN G T. Prognostics and health management of avionics[J]. Avionics Technology, 2009, 40(1): 30-38.

[5] ZHANG B ZH. Evolution and application of PHM technology[J]. Measurement \& Control Technology, 2008, 23(2): 5-7.

[6] ANDREW $\mathrm{K}$ S, LIN D, BANJEVIC D. A review on machinery diagnostics and prognostics implementing conditionbased maintenance[J]. Mechanical Systems and Signal Processing, 2006,20: 1483-1510. 
[7] XU P, KANG R. Research on prognostic and health management (PHM) technology[J]. Measurement and Control Technology, 2004,23(12): 58-60.

[8] MICHAEL G P.Prognostics and health management of electronics[M]. John Wiley \& Sons. Inc., Hoboken, New Jersey, 2008: 3-20.

[9] ANDREW H, LEO F. The joint strike fighter (JSF) PHM concept: Potential impact on aging aircraft problems $[\mathrm{C}]$, Proceedings of IEEE Aerospace Conference, Big Sky, Montana, USA, 2002,6: 3021-3026.

[10] PAN Q W, LI T, LI X SH. Research on the architecture of prognostics and health management system[J]. Journal of Electronic Measurement and Instrument, 2007 (Sup-pl.): 32-37.

[11] Hess A,Calvello G,Dabney T.[A].Aerospace Conference 2004 Proceedings.2004 IEEE[C]. 2001.

[12] Araiza M L, Kent R, Espinosa R.[A]. AUTOTESTCON Proceedings 2002 IEEE〔C〕 .Oct. 2002 Pages:818 - 841.

[13] Su L P, Nolan M, DeMare G, et al. [A]. AUTOTESTCON'99 IEEE Systems Readiness Technology Confer- ence [C]. 1999.

[14] Jay Lee,Fangji Wu,Wenyu Zhao,Masoud Ghaffari,Linxia Liao,David Siegel. Prognostics and health manage-ment design for rotary machinery systems-Reviews, methodology and applications $[\mathrm{J}]$. Mechanical Systems and Signal Processing, 2014,42(1-2).

[15] $\mathrm{Lu} \mathrm{Bo,} \mathrm{Lu} \mathrm{Yuping,} \mathrm{Fang} \mathrm{Xigao.} \mathrm{Research} \mathrm{on} \mathrm{Neural} \mathrm{Network}$ Dynamic Inverse Control of Hypersonic Vehi-cles[J]. Computer Measurement and Control, 2008, 16(7):966-968:

[16] Ma Ning, Lv Chen. Research on Aircraft Failure Prediction and Health Management Framework[ J]. Journal of Huazhong University of Science and Technology (Science and Technology), 2009, 37(Sup.): 207-209.

[17] Chang Qi, Yuan Shenfang. Aircraft Integrated Health Management (IVHM) System Technology Status and Development [J]. Systems Engineering and Electronics, 2009, 31(11): 2652-2657.

[18] Hu Changhua,Xu Hualong. Control system fault diagnosis and fault-tolerant control analysis and design [M]. Beijing: National Defense Industry Press, 2000.

[19] Hardman W, Hess A, Blunt D, et al. A USN development strategy and demonstration results for propulsion and mechanical systems diagnostics, prognostics and health management[ A]. Proceedings of the 2000 IEEE Ae-rospace Conference[ C]. Piscat-away: IEEE Inc., 2001: 3059-3068.

[20] Lv Chen. Fault Diagnosis and Prediction Technology - Principles and Applications [M]. [20] Beijing: Beijing University of Aeronautics and Astronautics Press, 2012

[21] Liu Zhen. Research on Intelligent BIT Diagnostic Method and Its Application in Multi-Electric Aircraft Pow-er System [D]. Xi'an: $\mathrm{PhD}$ thesis of Northwestern Polytechnical University, 2006
[22] Xu Lijia. Electronic system fault prediction and health management technology research [D]. Chengdu: Unv- ersity of Electronic Science and Technology PhD thesis, 2009.

[23] Liu Xiaohui.Study on RFID anti-collision algorithm based on binary number [D]. Changchun: Jilin University, 2015.

[24] Shuen Chih Tsai,Yu-Min Hu,Chen-Hsun Chai et al.Efficient tag reading protocol for large-scale RFID syst- ems with rereading[J]. Computer Communications, 2016,88:73-83.

[25] G. Eason, B. Noble, and I. N. Sneddon, "On certain integrals of Lipschitz-Hankel type involving products of Bessel functions," Phil. Trans. Roy. Soc. London, vol. A247, pp. 529-551, April 1955. (references)

[26] Zhang Bo. Research on Anti-collision Technology of CFD [D]. Shanghai: Donghua University, 2010.

[27] G. Eason, B. Noble, and I. N. Sneddon, "On certain integrals of Lipschitz-Hankel type involving products of Bessel functions," Phil. Trans. Roy. Soc. London, vol. A247, pp. 529-551, April 1955. (references)

[28] MICHAEL G P. Prognostics and health management of electronics[M]. John Wiley \& Sons.Inc., Hoboken, New Jersey, 2008, 3-20.

[29] VACHTSEVANOS G, LEWIS F, ROEMER M, et al. Intelligent Fault Diagnosis and Prognosis for Engineering Systems[M]. John Wiley \& Sons, Inc, 2006: 284-354.

[30] ADAMS D E. Nonlinear damage models for diagnosis and prognosis in structural dynamic systems[C]. Proceedings of SPIE Conference, 2002: 180-191.

[31] CHELIDZE D. Multimode damage tracking and failure prognosis in eletromechanical system[C]. Proceedings of SPIE Conference, 2002: 1-12.

[32] RAY A, TANGIRALA S. Stochastic modeling of fatigue crack dynamics for on-line failure prognostics[J]. IEEE Transactions on Control Systems Technology, 1996, 4(4): 443-451.

[33] LI Y, KURFESS T R, LIANG S Y.Stochastic prognostics for rolling element bearings[J]. Mechanical Systems and Signal Processing, 2000,14: 747-762.

[34] LUO J, BIXBY A, Pattipat I ,K,et al. An interacting multiple model approach to model-based prognostics[C]. in Proceedings of IEEE International Conference on System, Man and Cybernetics, Washington, DC, USA, 2003(1), 189-194.

[35] LESIEUTRE G A, FANG L, LEE U. Hierarchical failure simulation for machinery prognostics[C]. A Critical Link: Diagnosis to Prognosis, Virginia Beach, Virginia, USA, 1997, 103-110.

[36] FAN J Q, YAO Q W. Nonlinear time series: nonpara-metric and parametric methods[M]. USA: Springer, 2003, 10-27. 